\title{
OS QUESTIONÁRIOS DO SAEB: O QUE ELES REALMENTE MEDEM?
}

\author{
CAMILA AKEMI KARINO \\ LUÍS GUSTAVO DO AMARAL VINHA \\ JACOB ARIE LAROS
}

\section{RESUMO}

O presente estudo investigou as evidências de validade de construto dos questionários do Saeb de 2009. Depois de um processo de tratamento de dados e análise exploratória, foram analisadas as respostas de 1.906 .616 alunos do $9^{\circ}$ ano do ensino fundamental, de 77.905 professores e de 27.292 diretores, além das informações relacionadas às 27.045 escolas de todo Brasil. Análise Fatorial foi utilizada para identificar os construtos mensurados pelos questionários. Os resultados indicaram a existência de quatro fatores no questionário de escola, seis fatores no questionário de diretor, seis fatores no questionário de professor e quatro fatores no questionário de aluno. Uma reflexão crítica é necessária para avaliar se os construtos mensurados pelos questionários do Saeb são aqueles que realmente importam em relação ao desempenho escolar.

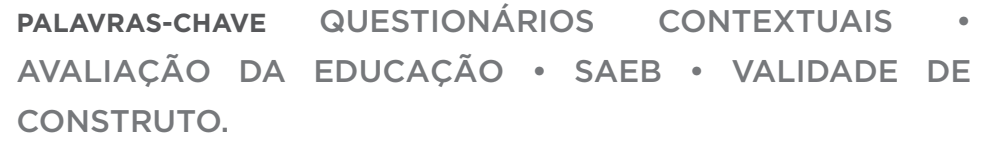




\section{RESUMEN}

El presente estudio investigó las evidencias de validez de constructo de los cuestionarios del Saeb del año 2009. Después del proceso de análisis exploratorio, fueron investigadas las respuestas de 1.906 .616 alumnos del $9^{\circ}$ año de enseñanza fundamental, de 77.905 profesores y de 27.292 directores, fuera las informaciones relativas a las 27.045 escuelas de todo el Brasil. La técnica de análisis factorial fue utilizada para identificar los constructos medidos por los cuestionarios. Los resultados revelaron a existencia de cuatro factores en el cuestionario de la escuela, seis factores en el cuestionario del director, seis factores en el cuestionario del profesor y cuatro factores en el cuestionario del alumno. Una reflexión crítica se hace necesaria para evaluar si los constructos medidos por los cuestionarios del Saeb son los que realmente interesan en relación al desempeño escolar.

\section{palabras clave cuEstionarios contextuales • EVALUACIÓN EDUCACIONAL • SAEB - VALIDEZ DE CONSTRUCTO.}

\section{ABSTRACT}

The present study investigated the evidence of construct validity of the Saeb questionnaires of 2009. After a process of data treatment and exploratory analysis, further analysis of the responses was carried out. The responses analyzed were from 1,906,616 students in the ninth grade, as well as of 77,905 teachers and 27,292 school directors, in addition to information related to 27,045 schools around Brazil. Factor analysis was used to identify the constructs measured by the questionnaires. The results indicated the existence of four factors in the school questionnaire, six factors in the director questionnaire, six factors in the teacher questionnaire and four factors in student questionnaire. Critical reflection is needed to assess whether the constructs measured by the Saeb questionnaires are those that really matter to school performance.

KEYWORDS CONTEXTUAL QUESTIONNAIRES • EDUCATION EVALUATION • SAEB • CONSTRUCT VALIDITY. 


\section{INTRODUÇÃO}

A promoção de educação a todos e de qualidade é objetivo de um país que busca ascensão. Nesse contexto, tornam-se essenciais o conhecimento das escolas que precisam de intervenção, a identificação de boas práticas e políticas públicas e a geração de estudos acerca dos fatores intervenientes na promoção de uma educação de qualidade. E o cerne da obtenção dessas informações está na realização periódica de boas avaliações do sistema educacional.

A avaliação tem como objetivo precípuo a geração de informação, no sentido tanto de apresentar um retrato da realidade quanto de subsidiar ações. Foi com o intuito de gerar informações sobre o desempenho e os resultados dos sistemas educativos que surgiram as avaliações externas no Brasil (PESTANA, 1998). Assim, a primeira avaliação externa instituída no Brasil, o Sistema de Avaliação da Educação Básica (Saeb), teve como principal objetivo oferecer subsídios para formulação, reformulação e monitoramento de políticas públicas que buscam a universalização do acesso e a ampliação da qualidade, da equidade e da eficiência da educação brasileira (BRASIL, 2007). 
Atualmente, esse sistema é constituído por três avaliações diferentes: Avaliação Nacional da Educação Básica (Aneb - amostral); Avaliação Nacional do Rendimento Escolar (Anresc); e Avaliação Nacional da Alfabetização (ANA) (BRASIL, 2013). A partir dessa nova constituição, o Saeb passa a monitorar a educação desde o $3^{\circ}$ ano do ensino fundamental até $o$ $3^{\circ}$ ano do ensino médio.

As avaliações externas são constituídas, essencialmente, por provas de avaliação do desempenho escolar e questionários que buscam avaliar fatores contextuais. Os questionários devem subsidiar a obtenção de informações acerca dos fatores contextuais que interferem na qualidade da educação e no desempenho escolar. Entretanto, que informações são essas? Quais são as informações coletadas? O que se busca medir por meio dos questionários? Diferentemente da delimitação e do aporte teórico que dão suporte à constituição das provas, não há tanta clareza sobre o que se pretende medir e o que se está medindo por meio dos questionários.

Considerando a necessidade de se discutirem os questionários do Saeb, instrumentos essenciais para a realização de uma boa avaliação, este estudo tem como objetivo analisar as evidências de validade de construto dos questionários aplicados na edição de 2009.

\section{A VALIDADE DE CONSTRUTO DOS QUESTIONÁRIOS DO SAEB}

A evidência da validade de construto é uma das principais características psicométricas de um instrumento de medida, ou seja, a evidência de que o instrumento está medindo realmente aquilo que se pretende medir. Essa característica é essencial, entre outras razões, por dois aspectos interligados à validade: utilidade e consequência do uso de um teste (URBINA, 2007).

Segundo Urbina (2007), a utilidade de um instrumento de medida está relacionada aos benefícios que ele traz para a tomada de decisões, sendo contingente na proporção em que o uso do instrumento promove uma maior precisão das inferências e decisões. Assim, a carência de evidências de validade do construto que está sendo medido tem implicações 
diretas acerca da utilidade de um instrumento de medida e, na ausência completa de evidências de validade, o teste torna-se inútil. Em conjunto à utilidade de um instrumento, deve-se pensar nas consequências individuais e sociais que o teste proporciona. A avaliação está relacionada a um julgamento de valor e se o termômetro desse julgamento não estiver medindo a essência do que se pretende medir, consequências negativas podem emergir.

Há pouca literatura acerca do que se pretende medir por meio dos questionários do Saeb e o que realmente eles estão medindo. Na maior parte das vezes, há informações gerais sobre o que os questionários buscam identificar: fatores contextuais; nível socioeconômico; aspectos culturais; etc. Todavia, verifica-se carência de uma definição clara e objetiva de cada um desses fatores, uma vez que eles podem representar uma série de informações. Há uma carência ainda maior de estudos que apresentem evidências de validade desses questionários.

Pestana (1998) menciona que os instrumentos do Saeb buscam avaliar quatro eixos: eficiência no ensino (medida por meio de provas de avaliação do desempenho); contexto (que engloba nível socioeconômico, perfil e autonomia das escolas); processo (que envolve planejamento e projeto pedagógico); e insumos (que incluem infraestrutura, instalações e equipamentos). Essa definição parece apontar algumas diretrizes a serem seguidas na construção dos instrumentos, mas ainda necessita de concepções teóricas mais claras e atualizadas.

O melhor aporte teórico acerca do que se pretende medir com os questionários do Saeb foi encontrado no documento Saeb 2001: Novas perspectivas (BRASIL, 2001) e no artigo de Franco et al. (2003). Nesse artigo, relata-se que a definição dos construtos a serem avaliados nos questionários teve como base teorias e resultados de pesquisas educacionais, especificamente: Lee, Bryk e Smith (1993); Willms (1992); Sammons, Hillman e Mortimore (1995); Mello (1994) e Brasil (1998). Com base nesses estudos, buscou-se priorizar na construção dos questionários do Saeb 2001: 
- seis construtos relacionados ao aluno - características sociodemográficas, capital social, capital cultural, motivação e autoestima, práticas de estudo e trajetória escolar;

- sete construtos relacionados à sala de aula - características sociodemográficas do professor, formação do professor, experiência profissional, condições de trabalho, estilo pedagógico, expectativa e miscelânea (que englobaria presença e rotatividade de professor e turno);

- treze construtos relacionados à escola - características sociodemográficas do diretor, formação do diretor, experiência do diretor, liderança, condições de trabalho do diretor e da equipe, trabalho colaborativo, organização do ensino e políticas de promoção, clima acadêmico, clima disciplinar, recursos pedagógicos, situação das instalações e equipamentos, atividades extracurriculares e miscelânea (que englobaria itens como admissão de alunos, critérios de formação de turmas, acesso aos resultados do Saeb, etc.).

Os autores esclarecem que esse referencial conceitual foi o melhor que pôde ser feito naquele momento e indicam a necessidade de investigações futuras que possibilitem aprimorar os instrumentos tanto em termos de linguagem quanto na definição de alguns construtos. Apesar de fazer referência aos instrumentos de 2001, o artigo ainda é atual considerando-se que pouco foi alterado nos questionários e que não há publicação desde então acerca da concepção teórica dos questionários.

Diante da relevância do Saeb no contexto brasileiro, algumas pesquisas já foram realizadas a fim de investigar a validade de construto de seus questionários. Em uma das pesquisas, por exemplo, foi feita uma análise fatorial utilizando os 163 itens relacionados à escola dos diferentes questionários do Saeb 2001 - Escola, Diretor, Turma, Professor e Aluno (RAUTER, 2003; RAUTER; LAROS; JESUS, 2007). Nessa pesquisa, foram encontrados 19 fatores: instalações físicas; liderança do diretor; recursos materiais e didáticos; 
comprometimento dos professores (avaliado pelo professor); comprometimento dos professores (avaliado pelo diretor); atitude positiva do alunado; clima disciplinar; formação continuada do diretor; experiência do diretor; TV e vídeo; segurança; vídeos didáticos; reforço escolar; recursos financeiros e pedagógicos; colaboração entre professores; equipe pedagógica; políticas contra repetências e abandono; estabilidade e assiduidade dos professores; e não ocorrência de roubos e depredações.

Todavia, é importante destacar que os autores mencionam as fragilidades psicométricas existentes em vários desses fatores. Além disso, Rauter (2003) aponta características dos questionários que dificultam a Análise Fatorial e que indicam problemas na construção dos questionários. Assim, a autora identificou como principais problemas dos questionários do Saeb 2001: a falta de simplicidade na formulação das perguntas (a questão deve ser formulada da forma mais objetiva possível - frases longas e detalhamentos desnecessários cansam a leitura), e a falta de precisão na formulação das perguntas (a questão deve medir apenas um único atributo). A autora observou também que os questionários do Saeb são muito extensos, o que pode comprometer a disposição para responder ao instrumento.

Outros estudos não tinham como foco principal a validade de construto dos questionários, mas buscavam encontrar evidências para fatores específicos, que, em geral, são utilizados em análises multinível para verificar o efeito escola. Andrade (2005), a fim de realizar uma análise dos fatores associados ao desempenho, procurou reduzir as variáveis dos questionários do Saeb 2001 a 11 fatores: nível socioeconômico; capital social; recursos culturais disponíveis em casa; experiência profissional dos professores; liderança do diretor; condições de trabalho do diretor e da equipe; trabalho colaborativo; clima acadêmico; clima disciplinar; recursos pedagógicos; e situações das instalações e equipamentos da escola.

Como aporte teórico, é importante também buscar referência nos questionários utilizados em avaliações externas estrangeiras e internacionais, tal como no Naep (Avaliação 
Nacional de Progresso Educacional) e no Pisa (Programa Internacional de Avaliação de Estudantes). No primeiro - um sistema de avaliação de abrangência nacional nos Estados Unidos -, são utilizados questionários aplicados a alunos, professores e escolas. Nos questionários dos alunos, são coletadas informações demográficas, nível socioeconômico, experiências em sala de aula e suporte educacional. Os professores são avaliados quanto a formação, treinamento e práticas pedagógicas. Por meio dos questionários das escolas, são avaliadas algumas características, tais como corpo discente, absenteísmo e evasão, corpo docente, práticas pedagógicas, recursos e envolvimento de pais e da comunidade (<http://nces.ed.gov/nationsreportcard/bgquest.aspx>).

Já o Pisa é uma iniciativa de avaliação internacional comparada que envolve estudantes de dezenas de países e economias. Na edição de 2009, o questionário respondido pelos estudantes buscava informações sobre características individuais, contexto familiar e recursos das casas, clima escolar, duração das aulas, acesso e uso de bibliotecas, gosto por leitura e estratégias de leitura e compreensão (essa edição tinha como domínio principal a leitura). O questionário das escolas visava avaliar a estrutura e organização, corpo docente e discente, recursos, currículos e avaliações, clima escolar, práticas e políticas educacionais, características do diretor ou responsável (ORGANIZATION FOR ECONOMIC CO-OPERATION AND DEVELOPMENT, 2012).

\section{MÉTODO}

\section{PARTICIPANTES}

Este estudo utilizou dados secundários coletados pelo Instituto Nacional de Estudos e Pesquisas Educacionais Anísio Teixeira (Inep) relativos à edição do Saeb realizada em 2009. Foram analisadas as respostas de estudantes, professores e diretores do $9^{\circ}$ ano do ensino fundamental de todo o país, além daquelas relacionadas às escolas. Foram consideradas escolas das diferentes dependências administrativas (federais, estaduais e municipais) e localizadas tanto nas cidades quanto no meio rural. 
A base de dados contém informações de 2.816 .856 alunos, variando de 13 a 1.405 alunos por escola. Das 32.343 escolas envolvidas no levantamento, $38,4 \%$ são estaduais e 62,5\% municipais; $83,4 \%$ são urbanas. Na distribuição entre as regiões, 9,5\% das escolas localizam-se no Norte, $34,3 \%$ no Nordeste, 33,1\% no Sudeste, $16 \%$ no Sul e 7,1\% no Centro-Oeste.

Também são disponibilizadas informações sobre professores e diretores. Neste estudo, foram utilizados os dados de 127.291 professores, dos quais $71,6 \%$ são mulheres e $67,9 \%$ possuem idade entre 30 e 49 anos. Entre os 32.344 diretores, 75,5\% são mulheres e 67,1\% têm idade entre 30 e 49 anos.

\section{INSTRUMENTOS}

Os instrumentos utilizados foram os questionários de contexto do Saeb. O questionário respondido pelos alunos é composto por 47 questões relacionadas, de modo geral, com o perfil do aluno, estrutura e itens presentes nas casas, escolaridade dos pais ou responsáveis, hábitos de leitura e incentivo dos pais aos estudos, trajetória e práticas escolares.

Os questionários de professores e diretores são compostos por 123 e 134 questões, respectivamente, e avaliam as condições de trabalho, práticas pedagógicas e de gestão, formação e experiência profissional, além de características sociodemográficas. As informações relacionadas com a infraestrutura das escolas são obtidas por meio do questionário de escola, composto por 66 itens, em que são avaliadas características como infraestrutura, estado de conservação e segurança (BRASIL, 2009).

\section{PROCEDIMENTOS}

Os questionários foram respondidos pelos participantes no mesmo dia em que foram aplicados os testes de conhecimento aos alunos. Os professores das disciplinas avaliadas, Língua Portuguesa e Matemática, e os diretores das escolas receberam os questionários antes do início das provas, devolvendo-os preenchidos ao final destas. Os alunos responderam ao questionário logo após a realização dos testes. Os questionários das escolas foram preenchidos por profissionais contratados pelo Inep. 


\section{ANÁLISE DE DADOS}

Os seguintes procedimentos de análise foram utilizados:

- análise exploratória dos dados - para verificação da integridade das bases de dados e da qualidade das respostas, foi realizada análise de dados missing e de normalidade univariada. A opção pairwise foi utilizada na Análise Fatorial para tratamento dos dados missing;

- Análise Fatorial - teve como base a Análise Fatorial dos eixos principais (Principal Axis Factoring, PAF), utilizando rotação Promax. Na Análise Fatorial foram excluídos itens que apresentaram cargas elevadas em mais de um fator e itens com carga fatorial no fator principal menor que 0,30 (LAROS, 2012; TABACHNICK; FIDELL, 2007);

- análise de fidedignidade - foi utilizado o coeficiente lambda $2\left(\lambda_{2}\right)$ de Guttman para estimar a fidedignidade dos fatores. Também considerou-se a correlação item-resto. Itens com correlação baixa entre o item e a escala total foram excluídos. Coeficientes de fidedignidade acima de 0,70 são considerados satisfatórios para a maioria das pesquisas básicas (KAPLAN; SACCUZZO, 2001; GEORGE; MALLERY, 2003).

As análises foram realizadas utilizando o software estatístico SPSS (Statistical Package for the Social Sciences), versão 18.

\section{RESULTADOS}

\section{ANÁLISE EXPLORATÓRIA DOS DADOS}

Durante as análises de consistência da base de dados, foram verificados percentuais altos de dados omissos. Dois tratamentos foram aplicados a todos os questionários: exclusão das variáveis com mais de 10\% de dados ausentes, e exclusão de casos com mais de $70 \%$ de dados ausentes. A Tabela 1 apresenta um resumo das características gerais dos questionários. 
TABELA 1 - Características dos questionários do Saeb 2009 - 9ao

\begin{tabular}{|c|c|c|c|c|c|}
\hline QUESTIONÁRIO & $\begin{array}{l}\text { TAMANHO DA } \\
\text { BASE DE DADOS } \\
\text { ORIGINAL }\end{array}$ & $\begin{array}{c}\text { TAMANHO DA } \\
\text { BASE DE DADOS } \\
\text { FINAL }\end{array}$ & $\begin{array}{l}\text { TOTAL DE } \\
\text { VARIÁVEIS }\end{array}$ & $\begin{array}{c}\text { № DE VARIÁVEIS } \\
\text { COM MAIS DE } \\
\text { 10\% DE MISSING }\end{array}$ & $\begin{array}{c}\text { № DE VARIÁVEIS } \\
\text { CONSIDERADAS } \\
\text { NA ANÁLISE } \\
\text { FATORIAL }\end{array}$ \\
\hline Escola & 32.002 & 27.045 & 67 & 15 & $51^{*}$ \\
\hline Professor & 127.291 & 77.905 & 121 & 68 & 53 \\
\hline Diretor & 32.344 & 27.292 & 134 & 48 & 86 \\
\hline Aluno & 2.817 .851 & 1.906 .616 & 47 & 9 & 38 \\
\hline
\end{tabular}

Fonte: Elaboração dos autores.

Nota $\left({ }^{*}\right)$ : Uma variável foi retirada da Análise Fatorial, pois consistia de uma percepção geral do avaliador acerca das condições da escola.

Nota-se que os questionários de escola e de aluno foram mais bem respondidos, uma vez que apresentam percentuais menores de variáveis com mais de $10 \%$ de dados ausentes (22\% e $19 \%$, respectivamente). Por outro lado, sobretudo no questionário de professor, destaca-se a quantidade de variáveis com mais de $10 \%$ de dados ausentes. A análise qualitativa das variáveis com percentual elevado de dados ausentes indicou problemas estruturais na formulação das perguntas (falta de simplicidade e de clareza, problemas nas opções de respostas, etc.), bem como a existência de perguntas comprometedoras ou passíveis de desejabilidade social. Além disso, esses resultados confirmam a existência de dificuldades na aplicação dos questionários de professor e diretor, seja pela logística adotada na coleta dos dados, seja pela falta de motivação dos agentes em responder aos questionários.

É preciso mencionar também que outras variáveis foram retiradas, pois tinham respostas qualitativas que não poderiam ser usadas numa análise quantitativa. Por exemplo, no questionário de diretor é perguntado como ele assumiu a direção da escola, se foi por processo seletivo, eleição ou indicação. Foram excluídas também variáveis como sexo, idade e etnia autodeclarada. Na análise do questionário de aluno, especificamente, não foram consideradas questões como gosto por Matemática e Língua Portuguesa, pretensões após o término do ensino fundamental, tipo de escola em que estudou, reprovação e abandono.

Considerando a relevância de uma boa qualidade da resposta nas análises subsequentes, julgou-se que seria melhor eliminar as variáveis com percentual acima de 10\% de dados 
ausentes. Dessa forma, optou-se por manter nos questionários somente as variáveis que foram adequadamente respondidas para, a partir delas, buscar definir o que parece medir os questionários do Saeb 2009.

Depois da exclusão das variáveis com mais de $10 \%$ de dados ausentes, a maioria das variáveis apresentava percentuais em torno de $2 \%$ de dados ausentes. No caso do questionário de escola, duas variáveis apresentaram excepcionalmente percentuais em torno de $5 \%$ e $8 \%$ de dados ausentes: quantidade-computadores e estrutura-biblioteca. Verificou-se que, na primeira variável, o avaliador externo precisava indicar o número total de computadores na escola, mas não havia opção a ser marcada caso a escola não tivesse computador $(\mathrm{n}=0)$. Logo, provavelmente o percentual de dados ausentes agregava também as escolas que não possuíam computador.

Para tratar essa variável, utilizou-se outra variável do questionário de escola que avaliava o estado de conservação dos computadores (conservação equipamentos-computador), na qual havia a opção "inexistente". Assim, nas escolas em que opção "inexistente" estava preenchida na variável conservação equipamentos-computador, os dados omissos da variável quantidade-computadores foram tratados como ausência de computador em vez de dado ausente. Após esse tratamento, restaram na base de dados somente $2,6 \%$ de respostas ausentes na variável quantidade-computadores.

Já a variável estrutura-biblioteca foi constituída a partir da inferência de uma variável do questionário de escola, pois não havia uma pergunta que avaliava diretamente a existência de uma biblioteca. Assim, a partir da pergunta: "Qual o percentual de alunos que utilizam a biblioteca durante o mês?”, desdobraram-se duas variáveis: frequência de uso da biblioteca pelos alunos e existência de biblioteca na escola, considerando que uma das opções de resposta era "não há biblioteca na escola". A primeira variável foi eliminada das análises por apresentar mais de 10\% de dados ausentes, enquanto a segunda, denominada estrutura-biblioteca, permaneceu nas análises mesmo com $8 \%$ de dados ausentes. Optou-se por manter a variável devido à relevância do quesito biblioteca para a infraestrutura escolar. 
Foram ainda realizados outros tratamentos a fim de se manter o maior número de variáveis possível. No questionário de diretor, um dos aspectos abordados está relacionado à ocorrência de agressões, questionando o tipo de agressão (física ou verbal), quem foi agredido (professor, funcionário ou aluno) e quem foi o agressor (professor, funcionário ou aluno), o que possibilitava um total de 18 respostas. De modo a diminuir o percentual de dados ausentes, foram considerados apenas os dois tipos de agressões para as três vítimas, independentemente do agressor. Já no questionário de aluno, uma variável única foi criada para relatar a existência de freezer em casa. Essa variável assumiu o valor 1 se o aluno respondeu que na sua casa tem freezer junto com a geladeira ou freezer separado da geladeira.

Após o tratamento das variáveis com quantitativo alto de dados ausentes, foi verificado se havia na base de dados casos (escolas, diretores, professores e alunos, a depender do questionário) com menos de $70 \%$ das questões respondidas. $\mathrm{O}$ intuito dessa análise era retirar os casos com muitas respostas faltantes. O questionário que apresentou maior redução no número de casos com base nesse critério foi o de aluno. De todo modo, considerando-se o tamanho da base de dados inicial, a exclusão dos casos com menos de 70\% de variáveis respondidas não compromete as análises posteriores e permite manter uma base com qualidade melhor das respostas.

\section{ANÁLISE FATORIAL E ANÁLISE DE CONSISTÊNCIA INTERNA}

O número inicial de fatores foi determinado pela análise do screeplot, do percentual de variância explicada por cada fator (acima de $2 \%$ ) e pelos autovalores (acima de 1) a partir de uma análise de componentes principais. Em seguida, executou-se uma sequência de análises fatoriais, no sentido de buscar uma solução considerando os fatores, as cargas fatoriais dos itens e a coerência de conteúdo dos itens e fatores.

As tabelas 2 a 5 apresentam as cargas fatoriais, as correlações item-resto e a fidedignidade por fator das análises dos questionários de escola, professor, diretor e aluno, respectivamente. 
TABELA 2 - Cargas fatoriais depois da rotação Promax (CF), correlações item-resto $\left(r_{i t}\right)$, fidedignidade dos fatores e a correlação entre os fatores do questionário de escola

\begin{tabular}{|c|c|c|}
\hline \multicolumn{3}{|c|}{ FATOR 1 - CONDIÇÃO DA ESTRUTURA } \\
\hline DESCRIÇÃO DO ITEM & $\mathrm{CF}$ & $\mathbf{R}_{\mathrm{IT}}$ \\
\hline Sala & 0,80 & 0,72 \\
\hline Parede & 0,74 & 0,67 \\
\hline Piso & 0,74 & 0,65 \\
\hline Estrutura elétrica & 0,68 & 0,65 \\
\hline Telhado & 0,67 & 0,57 \\
\hline Hidráulica & 0,67 & 0,68 \\
\hline Entrada da escola & 0,66 & 0,64 \\
\hline Portas & 0,66 & 0,66 \\
\hline Corredor & 0,65 & 0,62 \\
\hline Banheiro & 0,64 & 0,67 \\
\hline Pátio & 0,61 & 0,59 \\
\hline Cozinha & 0,60 & 0,60 \\
\hline Janelas & 0,59 & 0,61 \\
\hline Sala de aula arejada & 0,40 & 0,40 \\
\hline $\begin{array}{l}\text { Iluminação da sala } \\
\text { de aula }\end{array}$ & 0,35 & 0,36 \\
\hline Média (15 itens) & 0,63 & 0,61 \\
\hline Fidedignidade & 0,91 & \\
\hline
\end{tabular}

FATOR 2 - CONSERVAÇÃO DOS EQUIPAMENTOS

\begin{tabular}{l|c|c}
\hline DESCRIÇÃO DO ITEM & CF & $\mathbf{R}_{\mathbf{I T}}$ \\
\hline Telefone & 0,68 & 0,61 \\
\hline Fax & 0,63 & 0,56 \\
\hline $\begin{array}{l}\text { Quantidade de } \\
\text { computadores }\end{array}$ & 0,62 & 0,52 \\
\hline Impressora & 0,58 & 0,49 \\
\hline Retroprojetor & 0,56 & 0,49 \\
\hline Computador & 0,52 & 0,45 \\
\hline Som & 0,49 & 0,44 \\
\hline Máquina copiadora & 0,47 & 0,42 \\
\hline Projetor de slides & 0,43 & 0,40 \\
\hline Equipamentos contra & 0,42 & 0,43 \\
\hline incêndio & 0,40 & 0,38 \\
\hline Videocassete & 0,39 & 0,36 \\
\hline Televisão & 0,52 & 0,46 \\
\hline Média (12 itens) & 0,80 & \\
\hline Fidedignidade & & \\
\hline
\end{tabular}

Fidedignidade

\begin{tabular}{l:c:c}
\hline \multicolumn{2}{l}{ FATOR 3 - DEPREDAÇÃO } \\
\hline DESCRIÇÃO DO ITEM & $\mathbf{C F}$ & $\mathbf{R}_{\mathrm{IT}}$ \\
\hline Dependências internas & 0,75 & 0,67 \\
\hline Paredes & 0,70 & 0,61 \\
\hline $\begin{array}{l}\text { Dependências } \\
\text { externas }\end{array}$ & 0,69 & 0,61 \\
\hline Banheiro & 0,64 & 0,58 \\
\hline Pichação nos muros & 0,63 & 0,51 \\
\hline Sinais de depredação & 0,46 & 0,45 \\
\hline Média (6 itens) & 0,65 & 0,57 \\
\hline Fidedignidade & 0,81 & \\
\hline
\end{tabular}

\begin{tabular}{l|c:c}
\hline FATOR 4 - SEGURANÇA & \\
\hline DESCRIÇÃO DO ITEM & CF & $\mathbf{R}_{\mathrm{IT}}$ \\
\hline Vigia noite & 0,90 & 0,71 \\
\hline Vigia fim de semana & 0,73 & 0,62 \\
\hline Vigia dia & 0,53 & 0,47 \\
\hline Média (3 itens) & 0,72 & 0,60 \\
\hline Fidedignidade & 0,76 & \\
\hline
\end{tabular}

\begin{tabular}{|c|c|c|c|c|c|c|}
\hline $\begin{array}{l}\text { Correlação entre } \\
\text { fatores }\end{array}$ & $\begin{array}{c}\mathrm{F} 1-\mathrm{F} 2= \\
0,43\end{array}$ & $\begin{array}{c}F 1-F 3= \\
-0,42\end{array}$ & $\begin{array}{c}\mathrm{F} 1-\mathrm{F} 4= \\
0,06\end{array}$ & $\begin{array}{c}\mathrm{F} 2-\mathrm{F} 3= \\
-0,09\end{array}$ & $\begin{array}{l}F 2-F 4= \\
-0,08\end{array}$ & $\begin{array}{c}F 3-F 4= \\
-0,01\end{array}$ \\
\hline
\end{tabular}

Fonte: Elaboração dos autores 
$\mathrm{Na}$ análise do questionário de escola, quatro fatores foram encontrados (Tabela 2). Nota-se que a maior parte dos itens dos fatores, sobretudo dos dois primeiros, possui cargas fatoriais altas e correlações item-resto moderadas. Os coeficientes de fidedignidade dos quatro fatores identificados são satisfatórios. Esses resultados indicam uma boa estrutura fatorial e consistência interna adequada dos fatores. Vale destacar a existência de uma correlação moderada e positiva entre o fator 1 e o fator 2 e uma correlação moderada e negativa entre o fator 1 e o fator 3 . Ou seja, quanto mais positiva a percepção da estrutura, mais positiva tende a ser a percepção da qualidade dos equipamentos da escola, e menor a percepção de sinais de depredação. 
TABELA 3 - Cargas fatoriais depois da rotação Promax (CF), correlações item-resto $\left(r_{\text {it }}\right)$ e fidedignidade dos fatores do questionário de professor

\begin{tabular}{|c|c|c|c|c|c|c|c|}
\hline \multicolumn{4}{|c|}{ FATOR 1 - OCORRÊNCIA DE VIOLÊNCIA } & \multicolumn{4}{|c|}{$\begin{array}{l}\text { FATOR } 4 \text { - CAUSAS PROBLEMAS DE APRENDIZAGEM: } \\
\text { ESCOLA }\end{array}$} \\
\hline \multicolumn{2}{|c|}{ DESCRIÇÃO DO ITEM } & $\mathrm{CF}$ & $\mathbf{R}_{\mathrm{IT}}$ & \multicolumn{2}{|c|}{ DESCRIÇÃO DO ITEM } & $\mathrm{CF}$ & $\mathbf{R}_{\mathrm{IT}}$ \\
\hline \multicolumn{2}{|c|}{ Aluno com porte de arma de fogo } & 0,93 & 0,84 & \multicolumn{2}{|c|}{ Sobrecarga trab. dos professores } & 0,55 & 0,42 \\
\hline \multicolumn{2}{|l|}{ Atentado à vida } & 0,93 & 0,84 & \multicolumn{2}{|c|}{ Baixo salário dos professores } & 0,52 & 0,41 \\
\hline \multicolumn{2}{|c|}{ Professor vítima de roubo } & 0,92 & 0,82 & \multicolumn{2}{|c|}{ Carência de infraestrutura física/ped. } & 0,52 & 0,41 \\
\hline \multicolumn{2}{|l|}{ Agressão física } & 0,92 & 0,83 & \multicolumn{2}{|c|}{ Poucas oportunidades - desen. aluno } & 0,50 & 0,41 \\
\hline \multicolumn{2}{|c|}{ Aluno com porte de arma branca } & 0,84 & 0,77 & \multicolumn{2}{|c|}{ Insegurança física da escola } & 0,45 & 0,35 \\
\hline \multicolumn{2}{|c|}{ Professor vítima de furto } & 0,82 & 0,76 & \multicolumn{2}{|c|}{ Conteúdos curriculares inadequados } & 0,41 & 0,32 \\
\hline \multicolumn{2}{|c|}{ Alunos sob efeito de álcool } & 0,74 & 0,69 & \multicolumn{2}{|c|}{ Não cumprimento conteúdo curricular } & 0,38 & 0,30 \\
\hline \multicolumn{2}{|c|}{ Professor ameaçado por aluno } & 0,73 & 0,70 & \multicolumn{2}{|c|}{ Média (7 itens) } & 0,47 & 0,37 \\
\hline \multicolumn{2}{|c|}{ Alunos sob efeito de drogas ilícitas } & 0,70 & 0,66 & \multicolumn{2}{|c|}{ Fidedignidade } & 0,68 & \\
\hline \multicolumn{2}{|l|}{ Agressão Verbal } & 0,43 & 0,39 & & & & \\
\hline \multicolumn{2}{|l|}{ Média (10 itens) } & 0,80 & 0,73 & & & & \\
\hline \multicolumn{2}{|l|}{ Fidedignidade } & 0,92 & & & & & \\
\hline \multicolumn{4}{|c|}{ FATOR 2 - ATUAÇÃO DO DIRETOR } & \multicolumn{4}{|c|}{ FATOR 5 - EXPERIÊNCIA E CONDIÇÕES DE TRABALHO } \\
\hline \multicolumn{2}{|c|}{ DESCRIÇÃO DO ITEM } & $\mathrm{CF}$ & $R_{\mathrm{IT}}$ & DESCRIÇÃC & ITEM & $\mathrm{CF}$ & $R_{\text {IT }}$ \\
\hline Professor confia & no diretor & 0,88 & 0,80 & Professor-sa & bruto & 0,75 & 0,58 \\
\hline Diretor motiva & ara o trabalho & 0,86 & 0,79 & Professor-te & que está formado & 0,61 & 0,38 \\
\hline Diretor estimula & ativ. inovadoras & 0,84 & 0,78 & Professor-te & lecionando & 0,46 & 0,30 \\
\hline Comprometime & to diretor (prof.) & 0,79 & 0,75 & Professor-ca & horária & 0,43 & 0,38 \\
\hline Média (4 itens) & & 0,84 & 0,78 & Média (4 ite & & 0,56 & 0,41 \\
\hline Fidedignidade & & 0,90 & & Fidedignida & & 0,65 & \\
\hline $\begin{array}{l}\text { FATOR } 3 \text { - USO } \\
\text { PEDAGÓGICAS }\end{array}$ & DE MATERIAIS NAS & PRÁTI & & FATOR $6-F$ & MAÇÃO DO PROFESSO & & \\
\hline DESCRIÇÃO DC & ITEM & $\mathrm{CF}$ & $R_{\mathrm{IT}}$ & DESCRIÇÃC & ITEM & $\mathrm{CF}$ & $R_{\text {IT }}$ \\
\hline Computador & & 0,66 & 0,49 & Nível de esc & dade & 0,66 & 0,40 \\
\hline Vídeo-DVD & & 0,65 & 0,51 & Modalidade & urso superior & 0,64 & 0,43 \\
\hline Internet & & 0,63 & 0,46 & Natureza da & ituição em que formou & 0,56 & 0,36 \\
\hline Revistinhas & & 0,46 & 0,41 & Tipo de escc & o curso superior & 0,47 & 0,41 \\
\hline Livros de literat & & 0,44 & 0,38 & Média (4 ite & & 0,58 & 0,40 \\
\hline Jornais & & 0,44 & 0,39 & Fidedignida & & 0,58 & \\
\hline Média (6 itens) & & 0,55 & 0,44 & & & & \\
\hline Fidedignidade & & 0,73 & & & & & \\
\hline & $F 1-F 2=-0,04$ & F1-F & $=-0,02$ & $F 1-F 4=-0,03$ & $\mathrm{~F} 1-\mathrm{F} 5=0,01$ & 0,03 & \\
\hline $\begin{array}{l}\text { Correlação } \\
\text { entre fatores }\end{array}$ & $F 2-F 3=0,20$ & $F 2-\mathrm{F}$ & $=0,32$ & $\mathrm{~F} 2-\mathrm{F} 5=-0,02$ & $F 2-F 6=-0,04$ & $=0,22$ & \\
\hline & $F 3-F 5=0,19$ & F3-F & $=0,09$ & $F 4-F 5=0,10$ & $F 4-F 6=0,00$ & $=0,18$ & \\
\hline
\end{tabular}

Fonte: Elaboração dos autores. 
Os resultados da Análise Fatorial do questionário de professor (Tabela 3) demonstram, de modo geral, coerência de conteúdo dos itens com os fatores, uma estrutura fatorial aceitável e uma consistência interna razoável dos fatores. Os fatores 4, 5 e 6 apresentam consistência interna fraca (valores de $\lambda_{2}$ entre 0,58 e 0,68), mas optou-se por mantê-los porque esses resultados podem auxiliar no aprimoramento do questionário para aplicações futuras.

No questionário de professor, das 15 correlações entre fatores, nove têm valor menor que 0,10 . Nota-se, portanto, que em geral as correlações são baixas. Todavia, considerando que se trata de uma área pouco pesquisada, optou-se por apresentar as correlações, pois podem contribuir para o refinamento do instrumento. O mesmo ocorreu para os questionários de diretor e aluno. 
TABELA 4 - Cargas fatoriais depois de rotação Promax (CF), correlações item-resto $\left(r_{\text {it }}\right)$, fidedignidade dos fatores e a correlação entre os fatores do questionário de diretor

\begin{tabular}{|c|c|c|}
\hline \multicolumn{3}{|l|}{ FATOR 1- RECURSOS } \\
\hline DESCRIÇÃO DO ITEM & CF & $\mathbf{R}_{\mathrm{IT}}$ \\
\hline Internet para professores & 0,78 & 0,64 \\
\hline Computadores (professores) & 0,75 & 0,61 \\
\hline Internet para alunos & 0,73 & 0,58 \\
\hline Computadores para alunos & 0,66 & 0,53 \\
\hline Computadores (administrativo) & 0,58 & 0,54 \\
\hline Impressora & 0,56 & 0,52 \\
\hline Telefone & 0,53 & 0,59 \\
\hline Fax & 0,50 & 0,54 \\
\hline Biblioteca & 0,49 & 0,50 \\
\hline Aparelho de som & 0,48 & 0,49 \\
\hline Retroprojetor & 0,47 & 0,50 \\
\hline Fitas de vídeo ou DVD (laser) & 0,46 & 0,46 \\
\hline Quadra de esportes & 0,44 & 0,47 \\
\hline Vídeo cassete ou DVD & 0,43 & 0,42 \\
\hline Fita de vídeo/DVD (educativo) & 0,41 & 0,41 \\
\hline Laboratório & 0,41 & 0,38 \\
\hline Televisor & 0,41 & 0,40 \\
\hline Máquina copiadora & 0,39 & 0,38 \\
\hline Projetor de slides & 0,37 & 0,37 \\
\hline Média (19 itens) & 0,52 & 0,49 \\
\hline Fidedignidade & 0,88 & \\
\hline
\end{tabular}

FATOR 3 - EXPERIÊNCIA DO DIRETOR

\begin{tabular}{l:c:c}
\hline DESCRIÇÃO DO ITEM & CF & $\mathbf{R}_{\mathbf{I T}}$ \\
\hline Tempo como diretor & 0,91 & 0,65 \\
\hline Tempo de direção na atual escola & 0,79 & 0,59 \\
\hline Tempo da escolaridade atual & 0,40 & 0,40 \\
\hline Tempo de trabalho com educação & 0,39 & 0,43 \\
\hline Média (4 itens) & 0,62 & 0,52 \\
\hline Fidedignidade & 0,74 & \\
\hline
\end{tabular}

\section{FATOR 4 - FORMAÇÃO}

\begin{tabular}{l|c:c}
\hline Descrição do Item & CF & $r_{\text {it }}$ \\
\hline Área do título & 0,72 & 0,63 \\
\hline Título & 0,71 & 0,65 \\
\hline Forma de realização do curso & 0,54 & 0,35 \\
\hline Escolaridade & 0,51 & 0,40 \\
\hline Média (4 itens) & 0,62 & 0,51 \\
\hline Fidedignidade & 0,74 & \\
\hline
\end{tabular}

FATOR 5 - PARTICIPAÇÃO EM EVENTOS COM A COMUNIDADE

\begin{tabular}{l|c:c}
\hline DESCRIÇÃO DO ITEM & CF & $\mathbf{R}_{\mathbf{l T}}$ \\
\hline Mutirão: limpeza & 0,56 & 0,33 \\
\hline Mutirão: manutenção da estr. física & 0,54 & 0,34 \\
\hline Manutenção: hortas, pomar, jardins & 0,41 & 0,28 \\
\hline Camp. solidariedade: comunidade & 0,41 & 0,35 \\
\hline Eventos (terceiros) na escola & 0,35 & 0,30 \\
\hline Eventos da comunidade na escola & 0,33 & 0,29 \\
\hline Eventos da esc. para comunidade & 0,32 & 0,28 \\
\hline Camp. solidariedade: escola & 0,31 & 0,27 \\
\hline Média (8 itens) & 0,40 & 0,31 \\
\hline Fidedignidade & 0,62 & \\
\hline
\end{tabular}

(continua) 


\begin{tabular}{l:c:c}
\hline FATOR 2 - VIOLÊNCIA & CF & $\mathbf{R}_{\mathbf{I T}}$ \\
\hline DESCRIÇÃo DO ITEM & 0,67 & 0,57 \\
\hline Agressão verbal a alunos & 0,65 & 0,56 \\
\hline Agressão verbal a funcionários & 0,63 & 0,55 \\
\hline Agressão verbal a professores & 0,62 & 0,54 \\
\hline Agressão física a alunos & 0,49 & 0,42 \\
\hline Problemas disciplinares (alunos) & 0,37 & 0,34 \\
\hline Porte de arma branca & 0,36 & 0,35 \\
\hline Ação de gangues na escola & 0,54 & 0,48 \\
\hline Média (7 itens) & & \\
\hline Fidedignidade & & \\
\hline
\end{tabular}

\section{FATOR 6 - CARÊNCIAS RECURSOS HUMANOS}

\begin{tabular}{l|c:c}
\hline DESCRIÇÃO DO ITEM & CF & $\mathbf{R}_{\mathbf{I T}}$ \\
\hline Insuficiência de professores & 0,40 & 0,49 \\
\hline Carência de pessoal administrativo & 0,37 & 0,40 \\
\hline Rotatividade do corpo docente & 0,33 & 0,40 \\
\hline Alto índice de faltas (professores) & 0,32 & 0,38 \\
\hline Falta de apoio pedagógico & 0,30 & 0,28 \\
\hline Média (5 itens) & 0,34 & 0,39 \\
\hline Fidedignidade & 0,64 & \\
\hline
\end{tabular}

F1-F2 $=0,19$

$F 1-F 3=0,32$

F1-F4 $=0,33$

$\mathrm{F} 1-\mathrm{F} 5=0,08$

F1-F6 $=-0,05$

Correlação entre fatores

$\mathrm{F} 2-\mathrm{F3}=0,07$

$F 2-F 4=0,23$

$F 2-F 5=-0,08$

$\mathrm{F} 2-\mathrm{F} 6=0,02$

$\mathrm{F} 3-\mathrm{F} 4=0,27$

$\mathrm{F} 3-\mathrm{F} 5=0,03$

$F 3-F 6=0,00$

$\mathrm{F} 4-\mathrm{F} 5=0,1$

$F 4-F 6=-0,03$

$\mathrm{F} 5-\mathrm{F} 6=0,05$

Nota-se que a maior parte dos itens do questionário de diretor possui cargas fatoriais e correlações item-resto moderadas e índices de fidedignidade razoáveis. Os fatores 5 e 6 apresentam consistência interna fraca (valores de $\lambda_{2} 0,62 \mathrm{e}$ 0,64 , respectivamente). 
TABELA 5 - Cargas fatoriais depois de rotação Promax (CF), correlações item-resto $\left(r_{i t}\right)$, fidedignidade dos fatores e a correlação entre os fatores do questionário de aluno

\begin{tabular}{l:c:c}
\hline FATOR 1- NÍVEL SOCIOECONÔMICO & \multicolumn{1}{l}{ CF } & $\mathbf{R}_{\mathbf{I T}}$ \\
\hline DESCRIÇÃO DO ITEM & 0,74 & 0,60 \\
\hline Computador & 0,70 & 0,57 \\
\hline Internet & 0,64 & 0,57 \\
\hline Televisão em cores & 0,55 & 0,49 \\
\hline Carro & 0,53 & 0,48 \\
\hline Banheiro & 0,51 & 0,47 \\
\hline Lavadora de roupas & 0,45 & 0,41 \\
\hline Freezer & 0,44 & 0,40 \\
\hline Rádio & 0,40 & 0,38 \\
\hline Geladeira & 0,34 & 0,33 \\
\hline DVD & 0,53 & 0,47 \\
\hline Média (10 itens) & 0,80 & \\
\hline Fidedignidade & &
\end{tabular}

\begin{tabular}{l|c:c}
\hline \multicolumn{2}{l}{ FATOR 2 - DEVER DE CASA E CORREC̃ES } \\
\hline DESCRIÇÃO DO ITEM & CF & $\mathbf{R}_{\mathbf{I T}}$ \\
\hline Faz dever de Matemática & 0,67 & 0,46 \\
\hline Faz dever de Língua Portuguesa & 0,61 & 0,44 \\
\hline Prof. corrige dever de Matemática & 0,45 & 0,36 \\
\hline Prof. corrige dever de Português & 0,40 & 0,32 \\
\hline Professor elogia & 0,37 & 0,31 \\
\hline Média (5 itens) & 0,50 & 0,38 \\
\hline Fidedignidade & 0,63 & \\
\hline
\end{tabular}

\begin{tabular}{l|c:c}
\hline \multicolumn{2}{l}{ FATOR 3 - INCENTIVO DOS PAIS } & \\
\hline DESCRIÇÃO DO ITEM & CF & $\mathbf{R}_{\mathbf{I T}}$ \\
\hline Pais incentivam dever de casa & 0,69 & 0,53 \\
\hline Pais incentivam a estudar & 0,58 & 0,42 \\
\hline Pais incentivam a ler & 0,53 & 0,45 \\
\hline Pais incentivam a ir às aulas & 0,50 & 0,36 \\
\hline Média (4 itens) & 0,57 & 0,44 \\
\hline Fidedignidade & 0,65 & \\
\hline
\end{tabular}

\begin{tabular}{l:c:c}
\hline \multicolumn{2}{l}{ FATOR 4 - HÁBITO DE LEITURA DOS PAIS } \\
\hline DESCRIÇÃO DO ITEM & CF & $\mathbf{R}_{\mathbf{I T}}$ \\
\hline Vê mãe lendo & 0,70 & 0,38 \\
\hline Mãe sabe ler & 0,58 & 0,44 \\
\hline Pai sabe ler & 0,49 & 0,44 \\
\hline Vê pai lendo & 0,45 & 0,44 \\
\hline Média (4 itens) & 0,55 & 0,43 \\
\hline Fidedignidade & 0,66 & \\
\hline
\end{tabular}

\begin{tabular}{llll}
\hline Correlação entre fatores & $F 1-F 2=-0,09$ & $F 1-F 3=0,06$ & $F 1-F 4=0,34$ \\
& $F 2-F 3=0,25$ & $F 2-F 4=0,08$ & $F 3-F 4=0,24$ \\
\hline
\end{tabular}

Fonte: Elaboração dos autores.

A Análise Fatorial do questionário de aluno aponta uma estrutura fatorial aceitável, com resultados semelhantes aos dos demais questionários: cargas fatoriais e correlações item-resto moderadas e índices de fidedignidade razoáveis (Tabela 5). Os fatores 2, 3 e 4 apresentam consistência interna fraca (valores de $\lambda_{2}$ entre 0,63 e 0,66 ). 
Em todos os questionários, pode-se discutir a manutenção de alguns itens na composição dos fatores, todavia, considerando o caráter exploratório das análises e a intenção desse estudo de dar subsídios para o aprimoramento dos questionários, buscou-se manter a maior quantidade possível de itens.

Em resumo, na Análise Fatorial dos questionários do Saeb 2009, foi constatado que entre $45 \%$ e $71 \%$ dos itens não entraram na estrutura fatorial. O questionário com a maior proporção de itens excluídos foi o de professor (Tabela 6).

TABELA 6 - Resumo das variáveis excluídas nas análises por questionário

\begin{tabular}{c|c|c:c}
\hline QUESTIONÁRIO & TOTAL DE VARIÁVEIS & $\begin{array}{c}\text { TOTAL DE VARIÁVEIS } \\
\text { EXCLUÍDAS NAS ANÁLISES }\end{array}$ & $\begin{array}{c}\text { \% DE VARIÁVEIS } \\
\text { EXCLUIDAS DAS ANÁLISES }\end{array}$ \\
\hline Escola & 66 & 30 & $45 \%$ \\
\hline Professor & 121 & 86 & $71 \%$ \\
\hline Diretor & 134 & 86 & $64 \%$ \\
\hline Aluno & 47 & 24 & $51 \%$ \\
\hline
\end{tabular}

Fonte: Elaboração dos autores.

\section{DISCUSSÃO}

Em síntese, a Análise Fatorial dos questionários do Saeb 2009 resultou em quatro fatores no questionário de escola (qualidade da estrutura, qualidade dos equipamentos, depredação e segurança), seis no de professor (violência na escola, avaliação da atuação do diretor, uso de equipamentos nas práticas pedagógicas, experiência e condições de trabalho, fatores relativos à escola relacionados aos problemas de aprendizagem e formação do professor), seis no de diretor (recursos da escola, violência, experiência do diretor, formação do diretor e faltas e presença da comunidade) e quatro no de aluno (nível socioeconômico, lições e correções, incentivo e hábito de leitura dos pais).

Muitos fatores são convergentes com aqueles encontrados por Rauter (2003), uma vez que os questionários, embora sejam de edições diferentes (2001 e 2009), mantêm muitas similaridades. Além disso, diversos fatores estão relacionados 
com os eixos a serem avaliados pelos questionários segundo Pestana (1998): o eixo contexto engloba a percepção de violência de professores e diretores, a presença de equipes de segurança nas escolas e o nível socioeconômico dos alunos; o eixo de processo envolve os fatores relacionados com a percepção do professor quanto à atuação do diretor, as causas de problemas de aprendizagem atribuídos às escolas e aos alunos e o uso de equipamentos nas práticas pedagógicas, além do fator dever de casa e correções segundo a percepção dos alunos; e o eixo insumo contempla os fatores relacionados com a formação de professores e diretores, as condições de trabalho e qualidade de equipamentos e a infraestrutura.

Considerando a concepção teórica apresentada pelo Inep (BRASIL, 2001) e por Franco et al. (2003) acerca do que se pretende medir nos questionários do Saeb 2001, nota-se que nem todos os construtos foram contemplados nas análises realizadas neste artigo com os questionários de 2009. Entre os seis construtos relacionados ao aluno, observa-se que as características sociodemográficas são mensuradas e, no que concerne à Análise Fatorial, verifica-se que o capital social, o capital cultural e as práticas de estudo estão de certa forma contemplados. Motivação e autoestima e trajetória escolar não aparecem em nenhum dos fatores. Quanto aos construtos relacionados à sala de aula, os questionários do Saeb 2009 contemplam todos aqueles definidos em 2001, com exceção do construto miscelânea. Por fim, no que se refere aos construtos relacionados à escola, vários não são contemplados, tais como liderança, clima acadêmico, clima disciplinar, atividades extracurriculares e miscelânea (que englobaria itens como admissão de alunos, critérios de formação de turmas, acesso aos resultados do Saeb, etc.). Por outro lado, os construtos violência e segurança na escola aparecem em vários questionários, o que não estava previsto nos questionários de 2001.

Nota-se que construtos importantes estão sendo avaliados nos questionários do Saeb 2009. Todavia, ainda há fatores que precisam ser mais bem trabalhados, pois apresentam itens com baixas cargas fatoriais ou pouco itens. Vários fatores mostraram baixo coeficiente de fidedignidade: dos 
20 fatores identificados, oito tinham um coeficiente de fidedignidade inferior a 0,70. Além disso, há vários fatores associados ao desempenho dos alunos relevantes para a análise e que não são contemplados.

No Naep, por exemplo, os questionários respondidos pelos alunos apresentam itens relacionados com disciplinas, práticas pedagógicas, equipamentos utilizados e percepção de dificuldade de conteúdos e provas. Os professores das disciplinas avaliadas no Naep respondem a diversos itens referentes a formas de aperfeiçoamento e práticas pedagógicas utilizadas no dia a dia, enquanto no Saeb poucos itens associam-se a esses fatores, e na análise realizada, tais itens não se agrupam em fatores. Já o Pisa utiliza escalas no questionário dos estudantes que avaliam o clima escolar, as estratégias de aprendizagem e os hábitos de leitura, além da relação com o professor e práticas pedagógicas empregadas por estes. No Pisa, as escolas também são avaliadas por meio de escalas quanto à participação de professores nas decisões e ao envolvimento do diretor com a rotina da escola, aspectos esses também pouco abordados no Saeb.

Além das avaliações educacionais estrangeiras e internacionais, há estudos na área de fatores associados que indicam importantes aspectos que devem ser investigados no contexto brasileiro, pois interferem no desempenho (ANDRADE; LAROS, 2007; SOARES, 2007, 2005). A literatura tem mostrado a importância de fatores como capital social (BONAMINO et al., 2010; GOODMAN; GREGG; WASHBROOK, 2011), capital cultural (BONAMINO et al., 2010; MARTELETO; ANDRADE, 2013), atitudes e comportamentos dos alunos e pais (GABLE; WOLF, 1993; GOODMAN; GREGG; WASHBROOK, 2011; LEE, 2013), interações entre alunos e professores e clima escolar (STEWART, 2008; KONISHI et al., 2010), características de professores (WAYNE; YOUNGS, 2003) e liderança de diretores (LOUIS; DRETZKE; WAHLSTRON, 2010).

Ainda temos que nos perguntar sobre o que fazer com os itens que não entraram na Análise Fatorial. Cerca de 50\% dos itens dos questionários foram retirados das análises por apresentarem alto índice de respostas omissas ou durante a Análise Fatorial. Aparentemente esses itens não fazem parte 
de um construto, ou seja, com esses itens não é possível formar medidas com vários itens (multiple-item measures), sendo que em geral medidas com vários itens são melhores por serem mais fidedignas e terem mais evidências de validade.

Portanto, a partir deste estudo, verificou-se que, além de uma avaliação e definição clara dos construtos que se pretende medir, é necessária uma revisão na construção das questões dos questionários, pois várias não atendem às orientações para construção de instrumentos de medidas (PERES-DOS-SANTOS; LAROS, 2007; HOGAN, 2006; PASQUALI, 1999; CLARK; WATSON, 1995). Deve-se investir tempo na padronização das escalas das respostas, na construção de orientação para os pesquisadores quanto à possibilidade de utilização das variáveis como ordinais ou intervalares e na revisão das perguntas constantes nos questionários, de modo a torná-las mais simples e claras. Com essas ações, entende-se que os questionários tornar-se-iam mais eficientes.

Também é preciso investir em outras formas de coletas das informações. O uso de questionários em papel e a limitação das respostas a poucos professores têm reduzido a participação dos mesmos e inibido respostas sinceras devido ao medo de serem identificados. Assim, sugere-se o uso de questionários on-line com a participação de todos os professores das escolas, e não somente daqueles das séries avaliadas. É importante ainda um trabalho de conscientização sobre a importância dos questionários.

Ainda em relação a outras formas de coleta de informações, atualmente todos os participantes respondem a um questionário específico, o que gera uma enorme quantidade respostas acerca dos mesmos construtos. Para fins de pesquisa, trabalhar com um número maior de tipos de questionários pode ser mais eficiente, pois possibilitaria a coleta de informações de um número maior de construtos e cada participante responderia a um número menor de questões. Sabe-se que questionários muito longos tendem a não ser bem respondidos.

Entre as limitações deste estudo, é preciso apontar que as análises foram concentradas nas variáveis que poderiam ser trabalhadas de forma ordinal ou intervalar. Apesar de al- 
guns pesquisadores considerarem as variáveis ordinais com mais de dois valores como variáveis contínuas (PASQUALI, 2012b), sugere-se que outros estudos sejam realizados utilizando outros tipos de correlação. De todo modo, para os propósitos deste estudo, a análise fatorial empregada se mostra válida, pois permite apontar os fatores gerais que parecem ser medidos nos questionários do Saeb e gerar a reflexão sobre o que se pretende medir. O objetivo do artigo não foi criar medidas em si. Além disso, é preciso que outros estudos sejam realizados tendo como foco as variáveis nominais. Outra análise interessante que não foi realizada refere-se à verificação da reprodução dos construtos encontrados para o $9^{\circ}$ ano nos questionários do $5^{\circ}$ ano ou de edições posteriores do Saeb.

Por fim, espera-se que os resultados desta pesquisa possam motivar outros pesquisadores e apoiar o Inep a rever os questionários do Saeb a fim de que possam ser aprimorados. Os questionários dessa importante avaliação devem ser foco de contínuos debates sobre a pertinência dos referenciais teóricos, dos construtos almejados, das questões que compõem os questionários, dos indicativos de validade das medidas, enfim, da qualidade dos instrumentos como um todo.

\section{REFERÊNCIAS}

ANDRADE, Josemberg Moura de. Construção de um modelo explicativo de desempenho escolar: um estudo psicométrico e multinível com dados do SAEB. 2005. Dissertação (Mestrado em Psicologia Social e do Trabalho) - Instituto de Psicologia da Universidade de Brasília, Brasília, 2005.

ANDRADE, Josemberg Moura de; LAROS, Jacob Arie. Fatores associados ao desempenho escolar: estudo multinível com dados do SAEB/2001. Psicologia: Teoria e Pesquisa, v. 23, n. 1, p. 33-42, 2007.

BONAMINO, Alicia; ALVES, Fátima; FRANCO, Creso; CAZELLI, Sibele. Os efeitos das diferentes formas de capital no desempenho escolar: um estudo à luz de Bourdieu e de Coleman. Revista Brasileira de Educação, v. 15, n. 45, p. 487-499, 2010.

BRASIL. Instituto Nacional de Estudos e Pesquisas Educacionais. SAEB 2001: novas perspectivas. Brasília: Inep, 2001.

. SAEB - 2005 primeiros resultados: médias de desempenho do SAEB/2005 em perspectiva comparada. Brasília: Inep, 2007. 
. Microdados Prova Brasil 2009. Brasília: Inep, 2009. Disponível em: $<$ http://portal.inep.gov.br/basica-levantamentos-acessar>. Acesso em: 01 jun. 2012.

BRASIL. Ministério da Educação e do Desporto. Secretaria de Educação Fundamental. Referenciais para formação de professores. Documento Técnico. Brasília: SEF, 1998. 103p.

Portaria n. 482, de 7 de junho de 2013. Dispõe sobre o Sistema de Avaliação da Educação Básica - SAEB. Brasília: MEC, 2013.

CLARK, Lee Anna; WATSON, David. Constructing validity: basic issues in objective scale development. Psychological Assessment, v. 7, n. 3, p. 309-319, 1995.

FRANCO, Creso; FERNANDES, Cristiano; SOARES, José Francisco; BELTRÃO, Kaizô; BARBOSA, Maria Eugênia; ALVES, Maria Tereza Gonzaga. O referencial teórico na construção dos questionários contextuais do Saeb 2001. Estudos em Avaliação Educacional, n. 28, p. 39-74, 2003.

GABLE, Robert K.; WOLF, Marian B. Instrument development in the affective domain: measuring attitudes and values in corporate and school settings. 2. ed. Amsterdam: Kluwer Academic, 1993.

GEORGE, Darren; MALLERY, Paul. SPSS for Windows step by step: a simple guide and reference. 11.0 update. 4. ed. Boston: Allyn \& Bacon, 2003.

GOODMAN, Alissa; GREGG, Paul; WASHBROOK, Elizabeth. Children's educational attainment and behaviours of parents and children through childhood. Longitudinal and Life Course Studies, v. 2, n. 1, p. 1-18, 2011.

HOGAN, Thomas P. Introdução à prática de testes psicológicos. Rio de Janeiro: LTC, 2006.

KAPLAN, Robert M.; SACCUZZO, Dennis P. Psychological testing: principles, applications and issues. 5. ed. Belmont, CA: Wadsworth, 2001.

KONISHI, Chiaki.; HYMEL, Shelley.; ZUMBO, Bruno. D.; LI, Zhen. Do school bullying and student-teacher relationships matter for academic achievement? A multilevel analysis. Canadian Journal of School Psychology, v. 25, n. 1, p. 19-39, 2010.

LAROS, Jacob Arie. O uso da análise fatorial: algumas diretrizes para pesquisadores. In: PASQUALI, Luiz (Ed.). Análise fatorial para pesquisadores. Brasília: LabPAM, 2012. p. 141-160.

LEE, Jihyun. Can writing attitudes and learning behavior overcome gender difference in writing? Evidence from NAEP. Written Communication, v. 30, n. 2, p. 164-193, 2013.

LEE, Valerie E.; BRYK, Anthony S.; SMITH, Julia B. The organization of effective secondary schools. In: DARLING-HAMMOND, L. Review of Research in Education, v. 19, p. 171-267, 1993.

LOUIS, Karen Seashore; DRETZKE, Beverly; WAHLSTRON, Kyla. How does leadership affect student achievement? Results from a national US survey. School Effectiveness and School Improvement, v. 21, n. 3, p. 315-336, 2010. 
MARTELETO, Leticia; ANDRADE, Fernando. The educational achievement of Brazilian adolescents: cultural capital and the interaction between families and schools. Sociology of Education, v. 86, n. 4, p. 1-20, 2013.

MELLO, Guiomar Namo de. Escolas eficazes: um tema revisitado. In: XAVIER, Antonio Carlos da R. et al. (Org.). Gestão escolar: desafios e tendências. Brasília: Ipea, 1994. p. 329-369.

ORGANIZATION FOR ECONOMIC CO-OPERATION AND DEVELOPMENT. PISA 2009 Technical Report, PISA, OECD, 2012.

PASQUALI, Luiz. Instrumentos psicológicos: manual prático de elaboração. Brasília: LabPAM, 1999.

. Rotação dos fatores. In: PASQUALI, Luiz (Ed.). Análise fatorial para pesquisadores. Brasília: LabPAM, 2012a. p. 85-102.

. A correlação e a regressão. In: PASQUALI, Luiz (Ed.). Análise fatorial para pesquisadores. Brasília: LabPAM, 2012b. p. 85-102.

PERES-DOS-SANTOS, Luiz Francisco Bastos; LAROS, Jacob Arie. Avaliação da prática pedagógica do professor de ensino superior. Estudos em Avaliação Educacional, v. 18, n. 36, p. 75-95, 2007.

PESTANA, Maria Inês. O sistema de avaliação brasileiro. Revista Brasileira de Estudos Pedagógicos, v. 79, n. 191, p. 65-73, 1998.

RAUTER, Raíssa. Estrutura fatorial das questões do SAEB 2001 relacionadas a características da escola. Brasília, 2003, 124f. Dissertação (Mestrado em Psicologia Social e do Trabalho) - Instituto de Psicologia, Universidade de Brasília, Brasília, 2003.

RAUTER, Raíssa; LAROS, Jacob Arie; JESUS, Girlene Ribeiro de. Questionários contextuais do SAEB: análise qualitativa baseada nos resultados da Análise Fatorial. Revista Eletrônica Ibero-americana sobre Qualidade, Eficácia e Mudança em Educação-REICE, v. 5, n. 2, p. 56-68, 2007. Disponível em: <http://www. rinace.net/reice.htm>. Acesso em: mar. 2013.

SAMMONS, Pam; HILLMAN, Josh; MORTIMORE, Peter. Key characteristics of effective schools: a review of school effectiveness research. London: Office for Standards in Education OFSTED, 1995.

SOARES, José Francisco. O efeito da escola no desempenho cognitivo de seus alunos. In: MELLO E SOUZA, Alberto (Ed.). Dimensões da avaliação educacional. Petrópolis: Vozes, 2005. p. 174-204.

. Melhoria do desempenho cognitivo dos alunos do ensino fundamental. Cadernos de Pesquisa, v. 37, n. 130, p. 130-160, 2007.

STEWART, Endya B. School structural characteristics, student effort, peer associations, and parental involvement the influence of school-and individual-level factors on academic achievement. Education and Urban Society, v. 40, n. 2, p. 179-204, 2008. 
TABACHNICK, Barbara G.; FIDELL, Linda S. Using multivariate statistics. 5. ed. Boston: Pearson Education, 2007.

URBINA, Susana. Fundamentos da testagem psicológica. Porto Alegre: Artmed, 2007.

WAYNE, Andrew J.; YOUNGS, Peter. Teacher characteristics and student achievement gains: a review. Review of Educational Research, v. 73, n. 1, p. 89-122, 2003.

WILLMS, J. Douglas. Monitoring school performance: a guide for educator. Washington, D.C.: The Falmer, 1992.

\section{CAMILA AKEMI KARINO}

Mestre em Psicologia Social, do Trabalho e das Organizações pela Universidade de Brasília (UnB). Doutoranda do Programa de Pós-Graduação do Instituto de Psicologia da UnB, Brasília, Distrito Federal, Brasil camilaakarino@gmail.com

\section{LUÍS GUSTAVO DO AMARAL VINHA}

Mestre em Estatística pela Universidade de São Paulo (USP).

Professor Assistente do Departamento de Estatística da Universidade de Brasília (UnB), Brasília, Distrito Federal, Brasil lgvinha@gmail.com

\section{JACOB ARIE LAROS}

Doutor $\mathrm{PhD}$ em Psicologia pela University of Groningen, Holanda. Professor Associado do Instituto de Psicologia da Universidade de Brasilia (IP-UnB), Brasília, Distrito Federal, Brasil jalaros@gmail.com 